\title{
¿Por qué vale la pena randomizar en un estudio de terapia?
}

\author{
Blanca Peñaloza H, Roberto Candia Ba. \\ Why is it essential to randomize \\ a clinical trial on therapy?
}

$\mathrm{D}$ e acuerdo a lo presentado previamente en esta sección, los estudios clínicos randomizados (ECR) y las revisiones sistemáticas de ECR son el mejor diseño para responder preguntas relacionadas con screening o tamizaje y preguntas relacionadas con intervenciones terapéuticas ${ }^{1-4}$.

Los criterios para evaluar la validez interna de un ECR han sido definidos con claridad por varios autores ${ }^{3,5}$ :

1. ¿Fue la asignación de los pacientes a los grupos del estudio aleatoria (randomizada)? ¿Esta asignación fue oculta?

2. ¿Fue completo el seguimiento de los pacientes?

3. iFueron todos los pacientes analizados en los grupos a los que fueron asignados inicialmente (análisis por intención de tratar)?

4. ¿Fueron todos los participantes en el estudio ciegos a la intervención?

5. ¿Fueron los grupos en estudio tratados igual en todo menos en la intervención?

6. ¿Eran los grupos similares al inicio del estudio?

Los dos últimos criterios son menos importantes si se cumplen los cuatro primeros. En este artículo, analizaremos la relevancia y las características de la randomización.

\section{RELEVANCIA DE LA RANDOMIZACIÓN}

El principal objetivo de la randomización es evitar el sesgo de selección ${ }^{6}$. Existe sesgo de selección cuando en un estudio los grupos a comparar no son similares, especialmente en factores pronósticos que inciden en el evento evaluado (outcome). Esto puede causar que las diferencias en los resultados no puedan ser atribuidas a la intervención en estudio.

La randomización genera grupos comparables entre sí, tanto en factores pronósticos conocidos como desconocidos ${ }^{7,8}$, de tal forma que la única diferencia entre los grupos en estudio será la intervención.

Finalmente, la randomización garantiza la validez de los test de significación estadística ${ }^{9}$.

\section{CARACTERÍSTICAS DE LA RANDOMIZACIÓN EN UN ECR}

Para ser considerada adecuada, la randomización debe cumplir con 2 requisitos ${ }^{8,10}$ :

1. Generación de una secuencia de asignación de los pacientes a los grupos del estudio que sea al azar, impredecible.

Unidad de Medicina Basada en Evidencia y Departamento de Medicina Familiar, Pontificia Universidad Católica de Chile anterno de Medicina 70 año.

Correspondencia a: Dra. Blanca Peñaloza. E mail: bpenalo@med.puc.cl 
2. Ocultamiento de esta secuencia de asignación a los investigadores que distribuyen los pacientes hasta que el reclutamiento de éstos se haya completado ( «concealment of allocation»).

1. La generación de una secuencia de asignación de los pacientes al azar se refiere a que cada uno de los individuos incluidos en el estudio tenga una probabilidad conocida y frecuentemente idéntica de integrar el grupo control o el grupo experimental, en una secuencia de distribución determinada a través de un método aleatorio e imposible de predecir por los propios pacientes y el equipo investigador. Esto impide que, por ejemplo, la distribución de los pacientes incluidos en el grupo experimental sea manipulada y se produzca un grupo con factores pronósticos más favorables, que podrían producir un mejor resultado para la intervención en estudio ${ }^{2}$.

La generación de una secuencia de distribución se considera adecuada si disminuye el sesgo de selección al crear una secuencia por azar a través de métodos simples, como la asignación por lanzamiento de una moneda o dados, 0 métodos más complejos como números aleatorios producidos por programas computacionales 0 tablas de números aleatorios ${ }^{7,8}$.

La secuencia se considera inadecuada si se relaciona con factores pronósticos y, por lo tanto, es susceptible de introducir sesgo de selección: día, mes o año de ingreso, pacientes ingresados en horario hábil vs pacientes ingresados en horario nocturno.

2. Este punto se refiere a la necesidad de ocultar la secuencia de randomización. La ausencia de esta condición produce nuevamente riesgo de reclutamiento selectivo de los pacientes. Al conocer la secuencia de distribución, existe el riesgo que los investigadores encargados de reclutar, al conocer el grupo que le corresponderá a cada paciente, los rechacen en forma selectiva por considerarlos inapropiados»para el grupo asignado ${ }^{6-8}$, generando un sesgo de selección, a pesar del origen apropiado de la secuencia inicial. Por lo tanto, el ocultamiento protege la secuencia de asignación randomizada.

El ocultamiento se considera adecuado si ni los investigadores ni los pacientes pueden predecir la asignación de los pacientes. Esto se logra mediante: randomización centralizada (asignación desde un lugar distante del desarrollo del estudio); asignación de medicamentos de idéntico aspecto codificados previa y centralizadamente; utilización de sobres sellados opacos, idealmente numerados.

Métodos inadecuados de asignación son esquemas de asignación abiertos, por alternancia, números de ficha, o usando sobres abiertos o no opacos, que se pueden translucir ${ }^{8}$.

\section{LA EVIDENCIA DEL EFECTO DE LA RANDOMIZACIÓN} EN LOS RESULTADOS DE UNA INVESTIGACIÓN SOBRE TERAPIA

En la literatura médica existen numerosos ejemplos de la diferencia en el resultado de una misma intervención en estudios observacionales y en estudios randomizados. Un ejemplo de ello fue la recomendación de uso de estrógenos a mujeres posmenopáusicas en base a estudios observacionales, la cual ha cambiado después de la publicación de estudios randomizados que no demostraron tal beneficio ${ }^{11,12}$.

También existe evidencia de los efectos que la randomización inadecuada puede producir en los resultados de ensayos clínicos randomizados ${ }^{8,10}$.

Los estudios de Schulz et al ${ }^{10}$ y Moher et $\mathrm{al}^{13}$, muestran el efecto de una randomización inadecuada en los resultados de estudios clínicos randomizados. En ambos estudios, el ocultamiento inadecuado de la secuencia de asignación se asoció con una exageración del efecto terapéutico del 40\%, aproximadamente (Tabla 1). Estos resultados son confirmados por los hallazgos de la revisión sistemática de Juni et $\mathrm{al}^{8}$ que comparan el efecto de 3 variables metodológicas en los resultados de ECRs. La comparación de estudios metodológicamente adecuados con estudios inadecuados mostró que un ocultamiento inadecuado de la secuencia de asignación de pacientes se asocia a una exageración del efecto de la intervención en estudio. La medida de efectividad (odds ratio) de los estudios con ocultamiento inadecuado era en promedio 30\% más baja; es decir, los resultados de estos estudios mostraban mayor efectividad de las intervenciones que aquellos ensayos clínicos con ocultamiento adecuado.

En cambio, la generación de una secuencia de asignación inadecuada demostró tener un efecto 
Tabla 1. Resultados de 2 estudios empíricos que correlacionan aspectos metodológicos de los ECR y su efecto en sus resultados

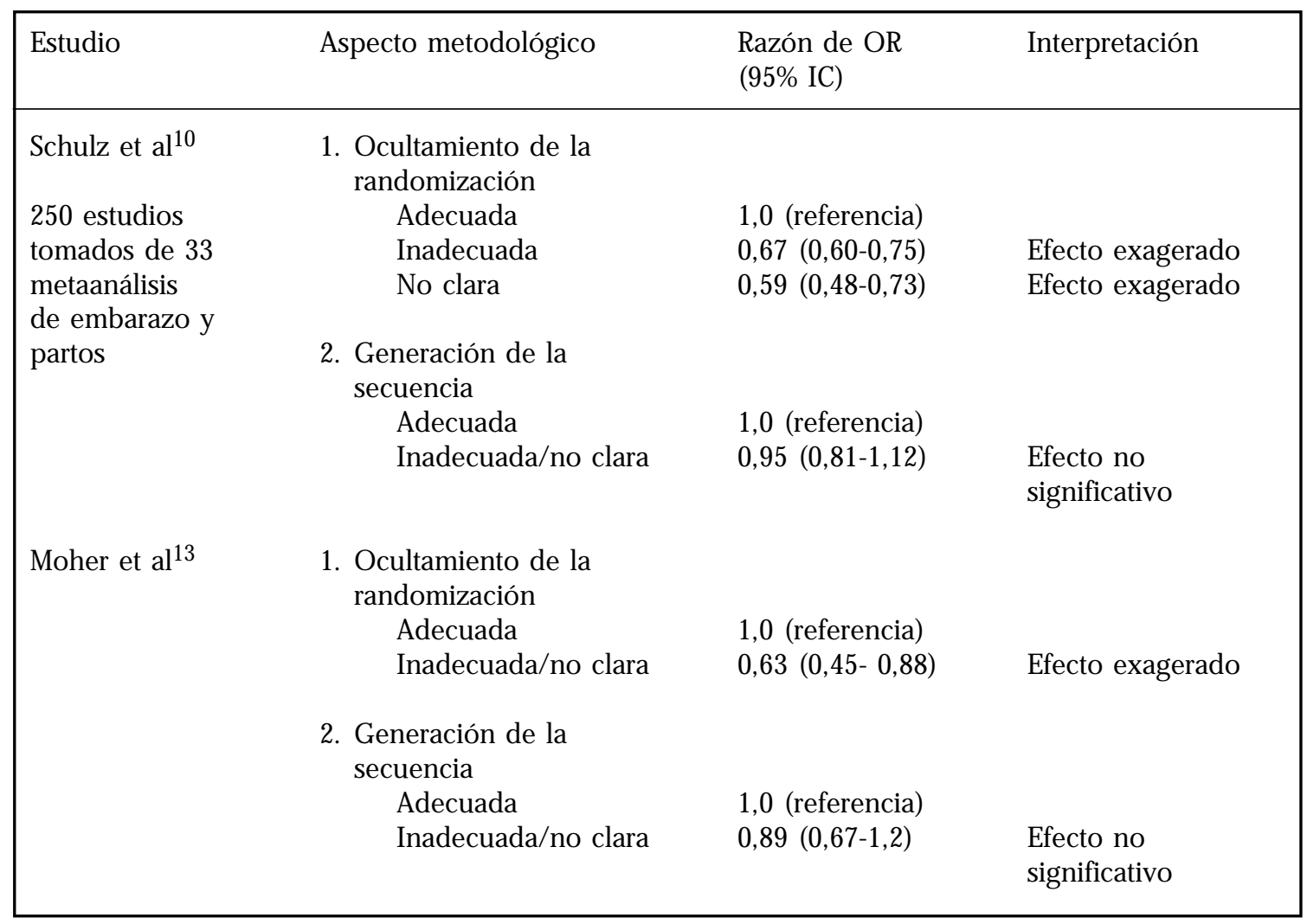

OR odds ratio. IC intervalo de confianza

no significativo en los resultados cuando se comparaban con estudios metodológicamente adecuados.

\section{¿CómO SABEMOS SI LA RANDOMIZACIÓN EN UN ECR ES ADECUADA?}

Una vez que el concepto de una randomización está claro y las características de sus dos etapas son conocidas, es relativamente fácil identificar los ensayos clínicos metodológicamente adecuados.

El lector interesado en realizar análisis crítico, debe considerar que no basta que los autores reporten «estudio randomizado»0 <estudio aleatorio», o estudio controlado»sino que deben hacer explícito el proceso de generación de la secuencia de randomización y la forma cómo se ocultó la secuencia de asignación a los investigadores a cargo de la selección de pacientes.

Así, la evaluación de la validez interna de un estudio está relacionada con la calidad del reporte de dicho estudio. Es decir, un reporte incompleto o poco claro puede corresponder a un estudio de metodología inadecuada ${ }^{10}$.

Se recomienda que la evaluación de la calidad de un aspecto metodológico se relacione con la calidad del reporte; por ejemplo, si un estudio reporta el método de randomización en forma incompleta o poco clara se debe considerar que probablemente corresponde a un estudio con deficiencias metodológicas ${ }^{2}$. 


\section{CONCLUSIONES}

- Los ECR o las revisiones sistemáticas de ECR se consideran el mejor diseño para investigar la eficacia de una intervención terapéutica. No obstante, deben ser sometidos a la evaluación de su validez interna que considera aspectos metodológicos como la randomización, variable que puede producir sesgo en los resultados de los estudios en cuestión.

- La randomización es uno de los aspectos metodológicos más importantes de un estudio que evalúa intervenciones terapéuticas. Para

\section{REFERENCIAS}

1. Sото M, Rada G. Formulación de preguntas en Medicina Basada en la Evidencia. Rev Méd Chile 2003; 131: 1202-7.

2. Egger M, Davey Smith G, Altman DG. Systematic Reviews in Health Care. Meta-analysis in context, Second ed. London: BMJ books, 2001.

3. Sackett D, Straus S, Richardson W. Evidence-based medicine. How to practice and teach EBM? Second ed. Edinburgh: Churchill Livingstone, 2000.

4. LeTelier LM, Moore P. La Medicina Basada en Evidencia. Visión después de una década. Rev Méd Chile 2003; 131: 939-46.

5. Guyatt G, Sackett D, Cook D. User's Guides to the Medical Literature. II. How to Use an Article about Therapy or Prevention? Are the results of the study valid? JAMA 1993; 270: 2598-601.

6. Schulz KF, KeNNETH K. Subverting randomization in controlled trials. JAMA 1995; 274: 1456-8.

7. AltMAn DG. Treatment allocation in controlled trials: Why randomize? BMJ 1999; 318: 1209.

8. Juni P, Altman DG, EgGer M. Systematic reviews in health care: Assessing the quality of controlled su evaluación se debe considerar si los pacientes incluidos en el estudio fueron distribuidos con un método aleatorio que asegure que su asignación fue al azar y en una secuencia impredecible y, por otra parte, que esta secuencia de distribución permaneció oculta hasta que se completó el reclutamiento de todos los pacientes incluidos en el estudio.

- De acuerdo a la evidencia disponible, una randomización inadecuada, especialmente el no-ocultamiento de la secuencia, produce resultados que exageran la efectividad de una terapia. clinical trials. BMJ 2001; 323: 42-6.

9. Byar DP, Simon R, Friedewald WT, Schesselman JJ, De Mets DL, Elennberg JH et al. Randomized clinical trials. N Engl J Med 1976; 295: 74-80.

10. Schulz KF, Chalmers I, Hayes RJ, Altman DG. Empirical evidence of bias. Dimensions of methodological quality associated with estimates of treatment effects in controlled trials. JAMA 1995; 273: 408-12.

11. Huley S, Grady D, Bush T, Furberg C, Hemington D, Riggs B ET AL. Randomized trial of estrogen plus progestin for secondary prevention of coronary heart disease in postmenopausal women. JAMA 1998; 280: 605-13.

12. Writing Group For The Women's Health Initiative INVESTIGATORS. Risks and benefits of estrogen plus progestin in healthy postmenopausal women: principal results from the women's health initiative randomized controlled trial. JAMA 2002; 288: 321-33.

13. Moher D, Pham B, Jones A. Does quality of reports of randomized trials affect estimates of intervention efficacy reported in meta-analyses? Lancet 1998; 352: 609-13. 